\title{
A programmable, multifunction dual-channel waveform generator for visual psychophysics
}

\author{
FREDERICK L. KITTERLE and ROBERT JONES \\ University of Toledo, Toledo, Ohio
}

\begin{abstract}
This paper describes a relatively low-cost, flexible means of presenting patterns on a CRT. The design allows for a number of options, such as presentation of gratings as well as nonstandard stimuli (white noise), the summation and differentiation of outputs to create complex luminance waveforms, and the switching of information from one channel to the other. In addition, the visual pattern generator permits a number of temporal options, such as phase reversals and the ramping on and/or off of a stimulus. This device incorporates a hardware means of scaling grating contrast. Finally, our procedure for producing synchronization signals, which uses internally generated interrupt signals, allows a number of software options, such as keyboard scanning and reaction timing, without significant time losses.
\end{abstract}

In this paper we describe a multifunction, programmable waveform generator to present stimuli on a CRT under computer control. A considerable number of psychophysical studies have used grating targets presented on a CRT to explore properties of the human visual system. Typically, these stimuli have been produced using the technique described by Campbell and Green (1965). Essentially, a very-high-frequency triangular signal $(100 \mathrm{kHz})$ from a waveform generator is applied to the vertical amplifier of an oscilloscope. The internal time base of the oscilloscope is used to drive the horizontal axis, usually at $50 \mathrm{kHz}$ or higher. A stationary vertical grating is produced by applying a signal to the $z$-axis of the oscilloscope and synchronizing it with the sweep of the horizontal axis. By varying the waveform (i.e., square wave, sine wave), the amplitude, and the frequency of the waveform generator that drives the $z$-axis amplifier of the oscilloscope, a square wave or sine wave grating that varies in contrast and spatial frequency can be produced. This technique may be modified so that the output of two or more function generators are used to drive the $z$-axis amplifier of a CRT. In this way, the response of the visual system to complex visual stimuli, formed by summing the luminance profiles of simple sinusoidal gratings that differ in spatial frequency, can be studied (Graham \& Nachmias, 1971). In addition, by varying the phase between the triggering and the modulating signals, gratings can be made to drift across the CRT at various rates (Graham, 1972).

All of the above functions can be performed under computer control (e.g., Arnold \& Hastings, 1984; Brown, 1977; Fritsch \& Keck, 1978; Rogers, 1976). For example, Fritsch and Keck described a relatively inexpensive means of producing gratings using a 6502-based microprocessor (KIM-1, MOS Technology). Although

Reprint requests should be sent to either author at the Department of Psychology, University of Toledo, Toledo, OH 43606. this technique is quite flexible and has been implemented in our laboratory, using a comparable microprocessor (SYM-1, Synertek Corp.), we have found a need for a device that incorporates features additional to those described by Fritsch and Keck.

Several of our experiments have involved discriminations in which subjects are required to determine whether two gratings differ in contrast, in spatial frequency, and/or in temporal frequency. Other experiments have explored the effect of an adapting grating on some property (such as apparent contrast) of a superimposed test grating. Thus, we desired a means of simultaneously or successively presenting similar or different gratings on two separate CRTs; a means of presenting gratings that can be phasereversed in time; a means of summing the output of two channels to produce a test and adapting grating, or combining the output of two channels to produce complex gratings; a means of varying the CRT on which a particular grating is presented over trials; and a means of implementing contrast scaling of gratings by hardware, rather than software, techniques.

The visual pattern generator we have developed was designed to meet the above specifications and incorporates the following features:

1. Its dual-channel capability allows either simultaneous presentation of similar visual stimuli or the presentation of two different stimuli on separate CRTs.

2. The design incorporates contrast control hardware, which allows for the contrast of a grating target to be scaled by a single instruction. This technique conserves memory in two ways. First, it avoids allocating computer memory for both a look-up or master table and a table of scaled values calculated from the master table to represent the contrast of the grating for a given trial. Second, memory is saved because a program is not necessary for scaling the values from the master table to produce a given contrast.

3. The addition of signal handling hardware permits in- 
terchannel summation and differentiation. For example, in the summation mode, one CRT can receive the output of a given channel (e.g., Channel B) while the other CRT receives the output of Channels $A$ and $B$. In addition, the outputs of the two channels can be interposed. Thus, the output on Channel A can be switched to Channel B and vice versa.

4. The design permits the contrast of the gratings to be phase-reversed in time. This can also be accomplished with the summated signals from both channels.

5. A 1-MHz oscillator provides a signal to the vertical amplifier of the CRT.

6. A frame clock provides a $50-100-\mathrm{Hz}$ signal.

The equipment used in our laboratory to present grating patterns includes a SYM computer with $32 \mathrm{~K}$ of memory and the programmable, multifunction, dualchannel waveform generator. The waveform generator, which is shown in Figure 1, contains five separate boards to implement visual pattern generation. (1) A logic interface (LI) board provides a hardware interface between two 8-bit versatile interface adapter (VIA) ports (VIA of the SYM microprocessor and a $50-100-\mathrm{Hz}$ frame generator). (2) A programming module (control logic interface, or CLI) with six control lines provides for computer control of Channel A and Channel B summation, interposition, and differentiation. These functions can also be implemented manually. (3) A dual digital-to-analog (DDAC) board provides digital-to-analog (D/A) conversion of the applied stimulus data. (4) A dual analog signal processor (DASP) board provides the necessary hardware for the implementation of interchannel summation, differentiation, interposition, and phase reversal. The board contains a 1-MHz vertical scan oscillator, which provides two buffered outputs for raster generation. (5) A power supply board provides the necessary voltage and the manual mode switches for manual mode operation. We have included a manual mode in this design so that features of the pattern generator may be selected manually, by setting dip switches, rather than by computer control.

The grating or waveform data for each CRT is stored in a master look-up table in computer memory. Similarly, contrast values are also stored in tables. Since we have a dual-channel design, there are separate tables for Channel A and B grating data and Channel A and B contrast data. These values are transferred to the waveform generator. The following discussion describes the processing

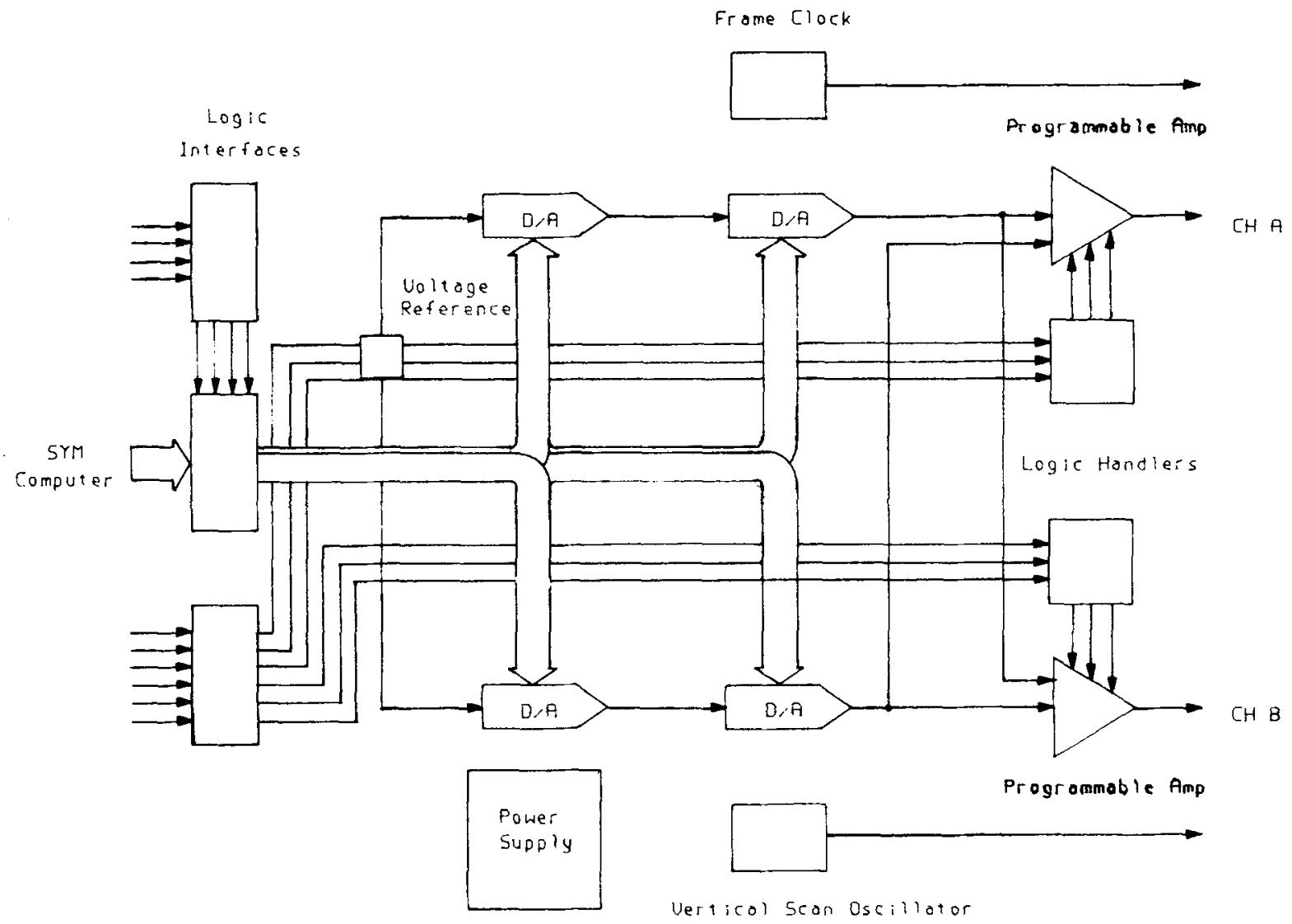

Figure 1. Overview of the programmable waveform generator. The five boards composing the generator are : Channel $A$ and Channel $B$ logic interfaces (LN), the dual digital-to-analog converter (DDAC), dual anlog-signal-processor (DASP)-programmable amplifiers, the control logic interface (CLI), and the power supply board. The vertical scan oscillator and the frame synchronizaton clock are also shown. 
of this data by each of the boards of the waveform generator. (We have excluded discussion of the power supply board.)

\section{BOARD}

This board serves as a logic interface to the SYM computer and is shown in Figure 2. In its present configuration, the board serves to buffer eight output lines of the APA port of the versatile interface adaptor (VIA \#2) on the SYM board. Four lines of the APB port of VIA-2 (PB5-7) are buffered and used as stimulus control lines to control the latching of grating data and contrast values. Additional lines (AAPA port) control summation, differentiation, and interchannel interposition. These functions are described later.

As mentioned earlier, our present approach utilizes an 8-bit bus structure. Channel $A$ and Channel $B$ grating data and contrast data are shared on a common 8-bit bus. It should be noted, however, that this approach can be altered. That is, future implementations could incorporate address decoders that would allow the pattern generator to be tied directly to the computer bus structure. Under this configuration, Channel A, Channel B, grating contrast, and function selection could be given unique addresses and treated as separate memory locations.

Channel A and Channel B data acquisition is controlled by the four APB control lines. Data sequencing is accom- plished by first loading the contrast value and strobing the appropriate contrast line. The operation need be done only once prior to a grating presentation and need not be done again until new contrast values are desired. Grating data is similarly loaded; however, the appropriate control line must be high for a load operation and low for a store operation. This allows for a transparent transfer mode, so that as grating data is presented on the bus, the data is immediately transferred to the analog processor (i.e., transparent latching). The LI board also contains two dip switches that permit manual setting of contrast levels.

After the latching and strobing operations are performed, the 8-bit data-bus information is transferred into four temporary latches. Two latches contain Channel A and Channel B grating data. The other two latches contain Channel A and Channel B contrast data. (There is flexibility in this design; the contrast values for one channel can be manually set while the values for the other channel can be set under program control.) The data is then transferred from the LI board via four 8-bit data buses to the DDAC board.

\section{DDAC BOARD}

The DDAC board, shown in Figure 3, receives the data from the LI board. Data transfers take place over four 8-bit data line sets. Two sets contain Channel A and Chan-

CH A Groting Lotch

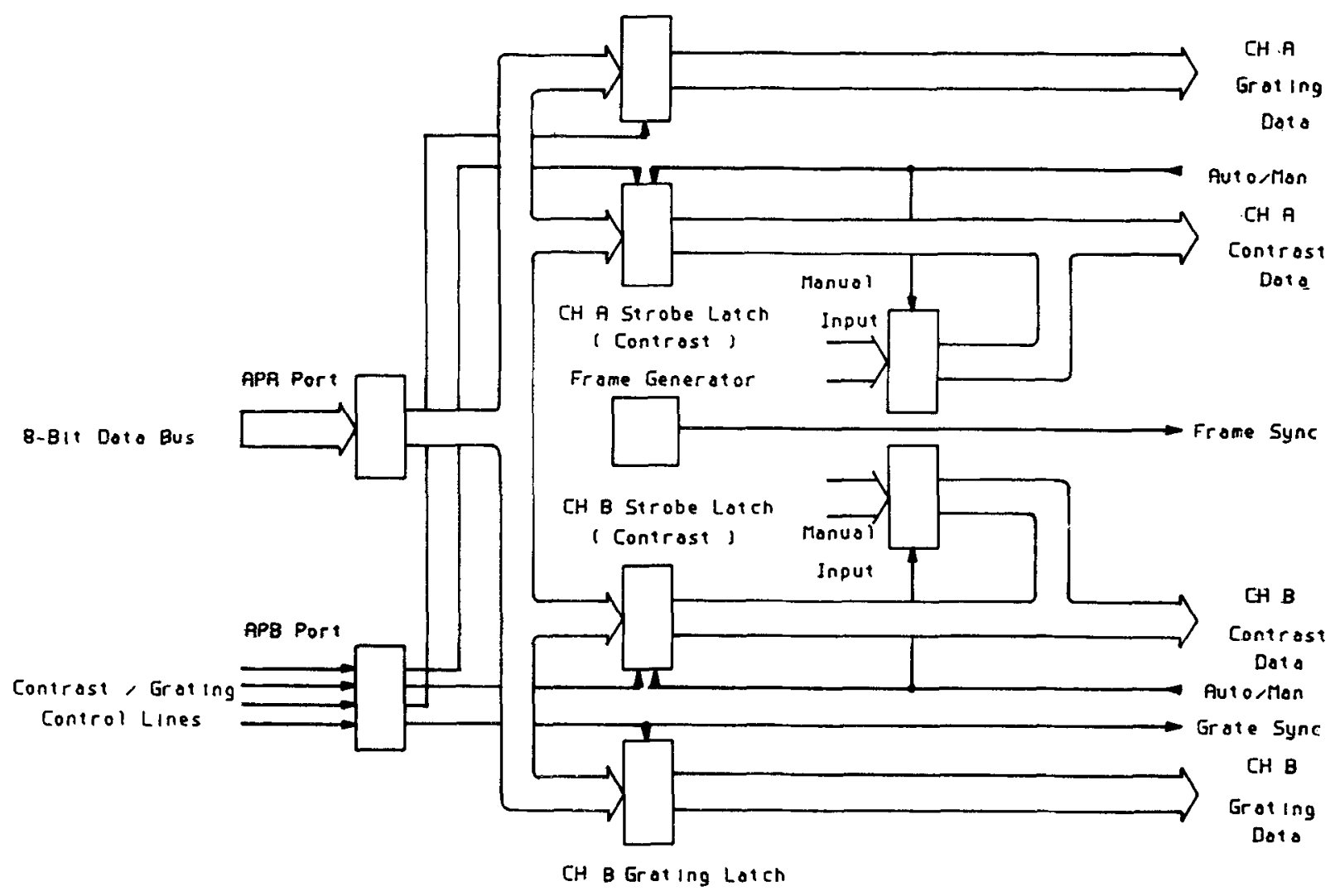

Figure 2. Logic interface board. The conceptual layout of this board for Channels A and B is shown (see text for details). 


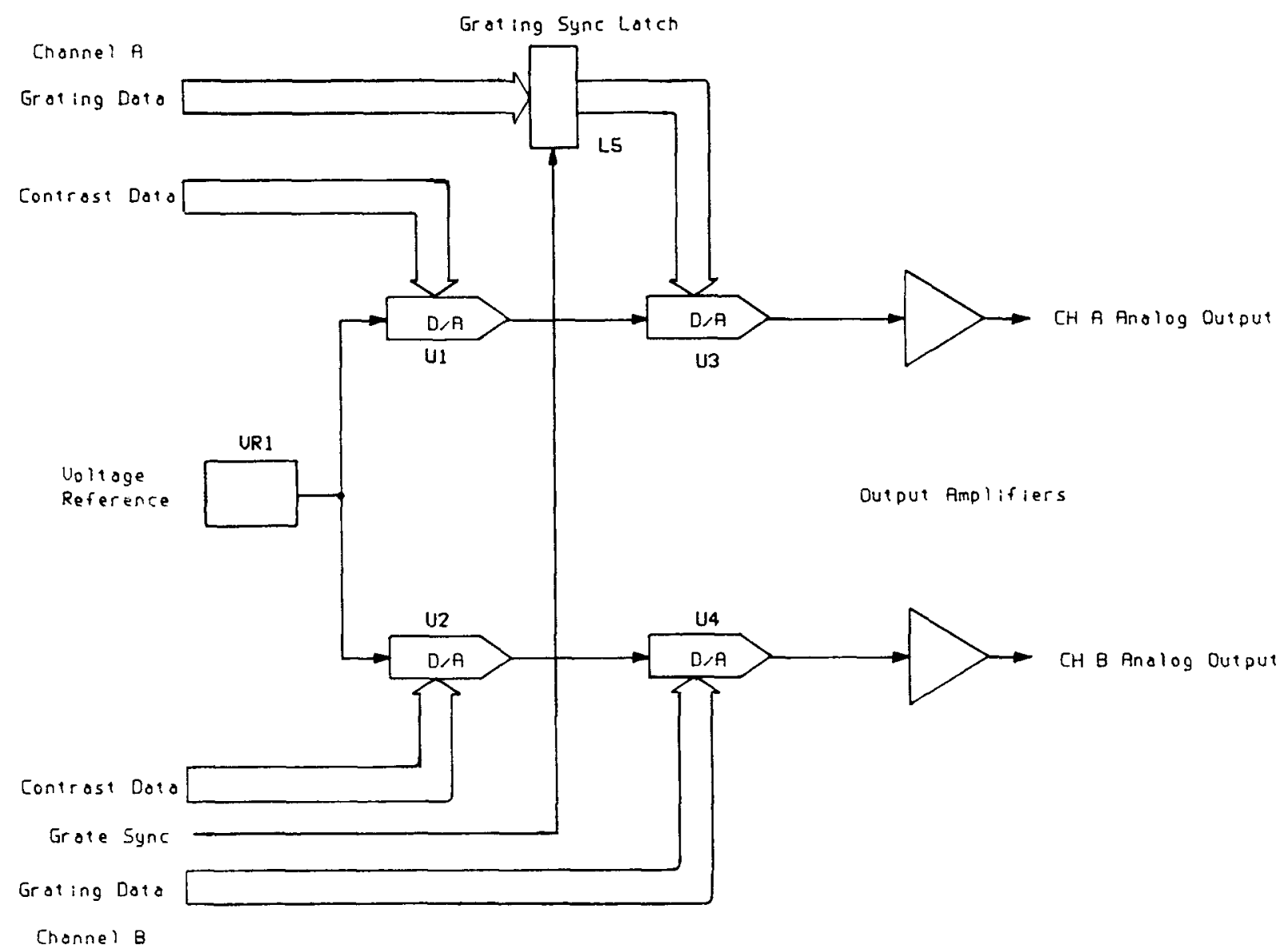

Figure 3. Dual digital-to-analog converter (DDAC) board. This figure shows the manner in which signals for Channels $A$ and B are processed by the system. U1 and U3 are the DACs for Channel A, and U2 and U4 are the DACs for Channel B. The function of the grating sync latch is discussed in the text.

nel B latched grating data and the other two contain Channel $A$ and Channel B latched contrast data. On-board processing of the received data is accomplished by a set of 8-bit multiplying D/A converters. As indicated in Figure 3, VR 1 provides the necessary temperaturecompensated reference voltages. This voltage $(2.00 \mathrm{~V})$, in turn, is fed to U1 and U2. These D/A converters, utilizing the latched contrast data, provide a processed or scaled reference voltage to $\mathrm{U} 3$ and $\mathrm{U} 4$. Contrast scaling is designed so that

$$
\mathrm{V}_{\text {contrast }}=\mathrm{V}_{\text {ref }} *(\text { Contrast Value/255), }
$$

with the possible range of contrast values being 0-255 (0-FF Hex). This scaling allows for 256 possible values of contrast. The contrast-scaled reference voltage is supplied as a scaling reference for the grating data D/A converters $\mathrm{U} 3$ and $\mathrm{U} 4$. The voltage function of these D/A converters is expressed by the formula

$$
\mathrm{V}_{\text {out }}=\mathrm{V}_{\text {contrast }} * \text { (Grating Data Value/255) }-1 \mathrm{~V} \text {. }
$$

The major utility of providing hardware scaling of contrast is that scaling does not adversely affect the quality or resolution of the waveform produced by our device.
Also, as new contrast values are selected, they may be implemented by simply strobing in those values.

Note that in the design of this board there is a component labeled "Grating Sync Latch" (GSL) (see Figure 3). This component is necessary in the generation of a complex grating produced by the summation of the outputs of Channel A and Channel B. During normal operation, Channel $\mathbf{A}$ and Channel $B$ grating data are sequentially latched, scaled, and generated by D/A converters 1-4. These output voltages are then passed to the DASP board, which provides the various functions of interchannel summation, differentiation, interposition, and phase reversals. However, these functions are performed in an analog manner and in real time. Consequently, a definite time slew will exist between the time when the Channel A grating value has been latched and the time when the Channel $B$ grating value has been latched on the LI board. The GSL resolves the problem of time slew in the following way: The value of Channel A from the LI board will be phasedelayed by the GSL. This information is held until the same control signal that is used to latch Channel B data also enables the GSL. When this occurs, the grating data on Channel A and Channel B will be simultaneously 
presented to their appropriate D/A converters (i.e., U3 and U4, respectively). This interdependence between Channel A and Channel B provides the logic necessary to avoid problems associated with time slewing.

There may be times when we may wish to bypass this feature and operate Channels A and B independently. For example, this would be the case if we wished to use Channel $B$ to present a mean luminance level and Channel A to present a grating. To provide this flexibility, the GSL on the DDAC board can be disabled. If this option is selected, the waveform generator cannot be used to properly utilize the features of summation and differentiation.

\section{DASP}

Information received from the DDAC board is processed by the DASP board. This board, which is shown in Figure 4, allows for the following functions to be performed:

Interchannel Summation

$$
\begin{aligned}
\mathrm{CH} \mathrm{A}_{\text {out }} & =\mathrm{CH} \mathrm{A}+\mathrm{CH} \mathrm{B} \\
\mathrm{CH} \mathrm{B} \mathrm{B}_{\text {out }} & =\mathrm{CH} \mathrm{B}+\mathrm{CH} \mathrm{A}
\end{aligned}
$$

Interchannel Differentiation

$$
\begin{aligned}
\mathrm{CH} \mathrm{A}_{\text {out }} & =\mathrm{CH} \mathrm{A}-\mathrm{CH} \mathrm{B} \\
\mathrm{CH} \mathrm{B} \mathrm{B}_{\text {out }} & =\mathrm{CH} \mathrm{B}-\mathrm{CH} \mathrm{A}
\end{aligned}
$$

Interposition Selection

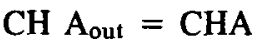

$$
\begin{aligned}
& \mathrm{CH} \mathrm{A}_{\text {out }}=\mathrm{CH} \mathrm{B} \\
& \mathrm{CH} \mathrm{B} \mathrm{B}_{\text {out }}=\mathrm{CH} \mathrm{B} \\
& \mathrm{CH} \mathrm{B}_{\text {out }}=\mathrm{CH} \mathrm{A}
\end{aligned}
$$

Phase Reversal

$$
\begin{aligned}
& \mathrm{CH} \mathrm{A}_{\text {out }}=\text { Option } 1,2,3 \text { phase reversed } \\
& \mathrm{CH} \mathrm{B}_{\text {out }}=\text { Option } 1,2,3 \text { phase reversed }
\end{aligned}
$$

We shall discuss the conceptual logic of how analog data is handled at this level of data processing.

As shown in Figure 4, the DASP board contains two steering logic handlers, which provide the necessary logic commands to the function amplifiers. The steering logic handlers receive two 3-bit command signals from the DASP logic controller interface (LCI). This board is discussed in more detail below. Each 3-bit signal provides four possible command states or functions. Two of the

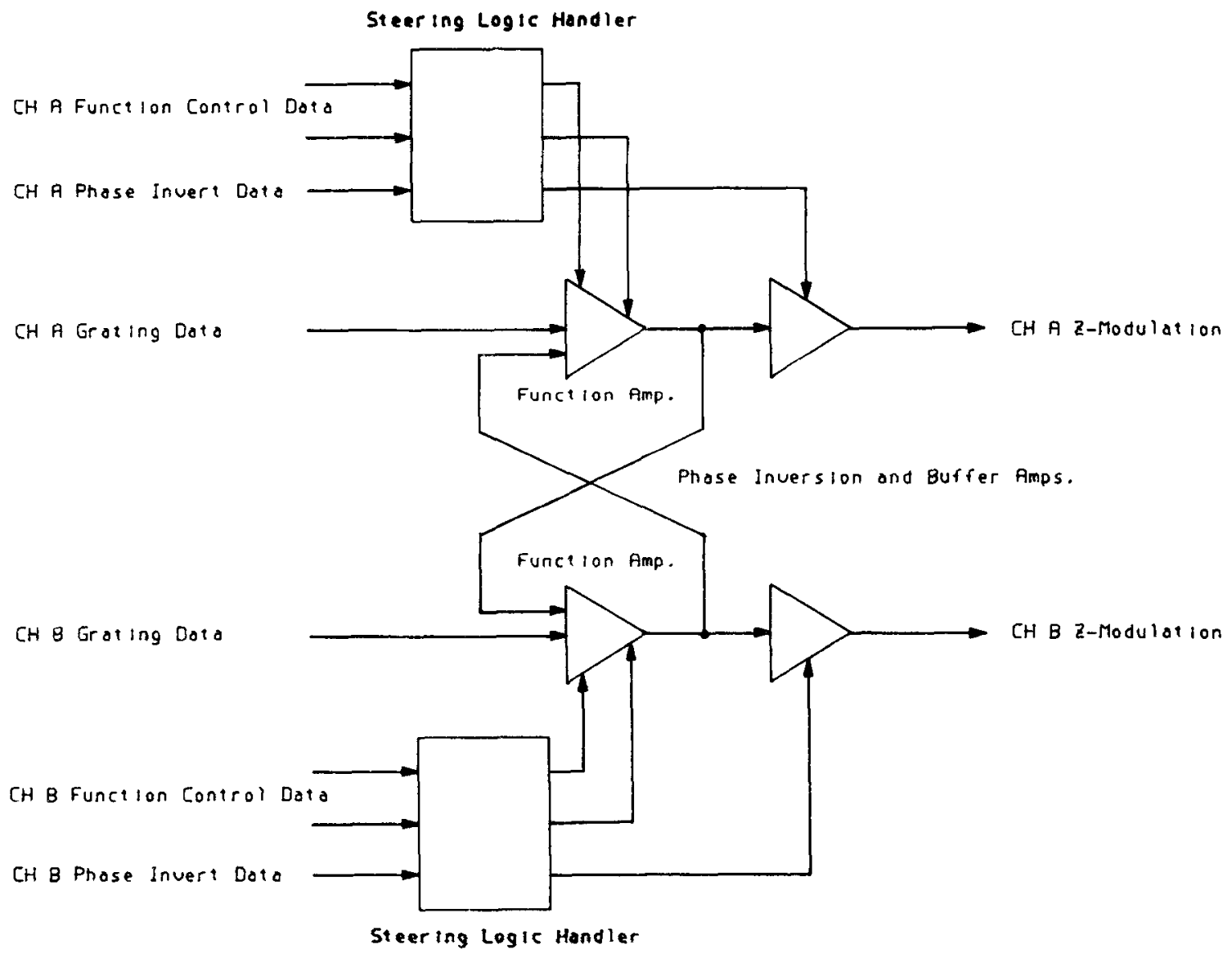

Figure 4. The dual analog signal procescor. Control lines to the steering logic handlers for Channels A and B are shown. The center of the figure shows the function amplifies for summetion, differentiation, and interpocition of Channd A and Channal B stands. 
3 bits are used to select either the sum or difference states, as well as channel interposition. The third bit is reserved for phase-reversal operations. The 2-bit command sequence of the 3-bit signal, as directed by the DASPs' logic handlers, programs the connection of analog switches to an operational amplifier. By properly addressing these switches, the various channel inputs can be obtained; selection of one of the three possible functions or modes is accomplished through the configuration of the operational amplifier with these switches. The various configurations are shown in Figures 5-8.

In Figures 5 and 6, both channels are in the same configuration or functional mode. In Mode 1 (Figure 5a), the switch combination configures channel inputs to be added together (summation). In Mode 2 (Figure 6), the channel inputs are subtracted from one another (differentiation). In Mode 3, channel inputs are either passed directly (Figure 7) or interchanged (i.e., interposed) (Figure 8).

After signal processing by the DASP, the channel information is passed through an additional operational amplifier. The configuration is presented in Figure 9, in which Channel $\mathrm{A}$ is shown as noninverting and Channel $\mathrm{B}$ as inverting. The 3-bit control signal directs the operation of a phase-inversion switch, which controls grating contrast reversals. If the switch is enabled (closed), the amplifier inverts the signal (i.e., a phase reversal occurs, assuming the waveform is symmetrical about the $x$-axis). Conversely, if the switch is disabled (open), the amplifier acts as a unity gain follower. From the output of this board, the information is passed to the $z$-modulation input of the display oscilloscope.

Included on this board is a $1-\mathrm{MHz}$ vertical scan oscillator that generates a bipolar square wave signal of approximately $\pm 5 \mathrm{~V}$ (see Figure 1). It is of a free-running type and is designed to provide a uniform high vertical scan frequency as an input to the vertical amplifier of a CRT. At a nominal $20-\mathrm{msec}$ grating presentation over a $5^{\circ}$ field of vision, this amounts to approximately 40,000 vertical raster lines to be generated. Given a 512-grating data field, this amounts to approximately 78 raster lines per data point, or roughly 8,000 raster lines per degree of visual field.

A few comments about the design should be mentioned at this point. All of the functions described occur in real time; therefore, at any time during the presentation of a grating, any of the above modes may be changed-even during a frame. This implies that grating phase reversal could occur several times during the actual grating presentation. Similarly, channels could be interposed during this same interval. Contrast can also be changed during a presentation. Because contrast can be changed quite

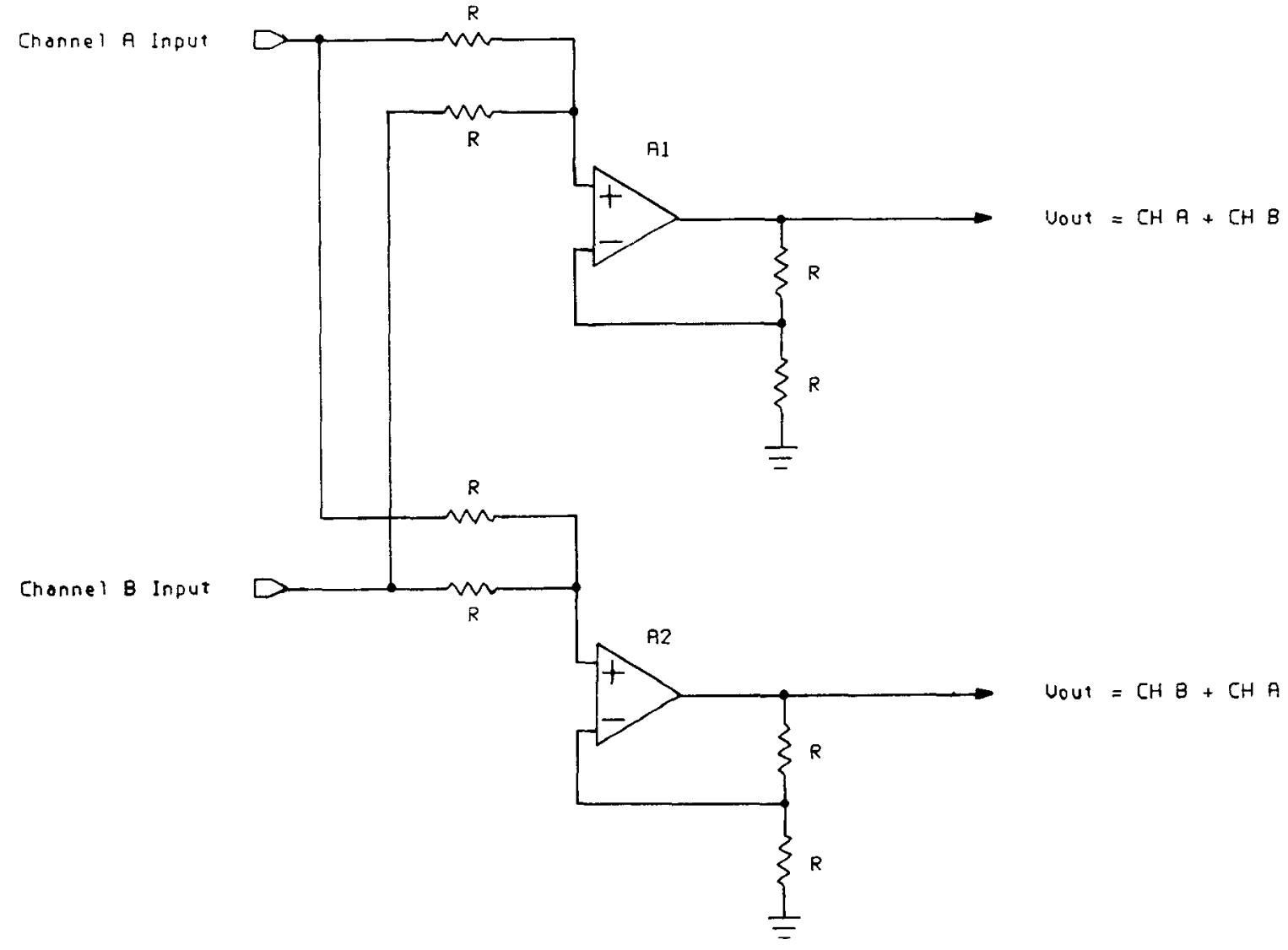

Figure 5. Function amplifiers configured for summation. 
Chonnel fo Input

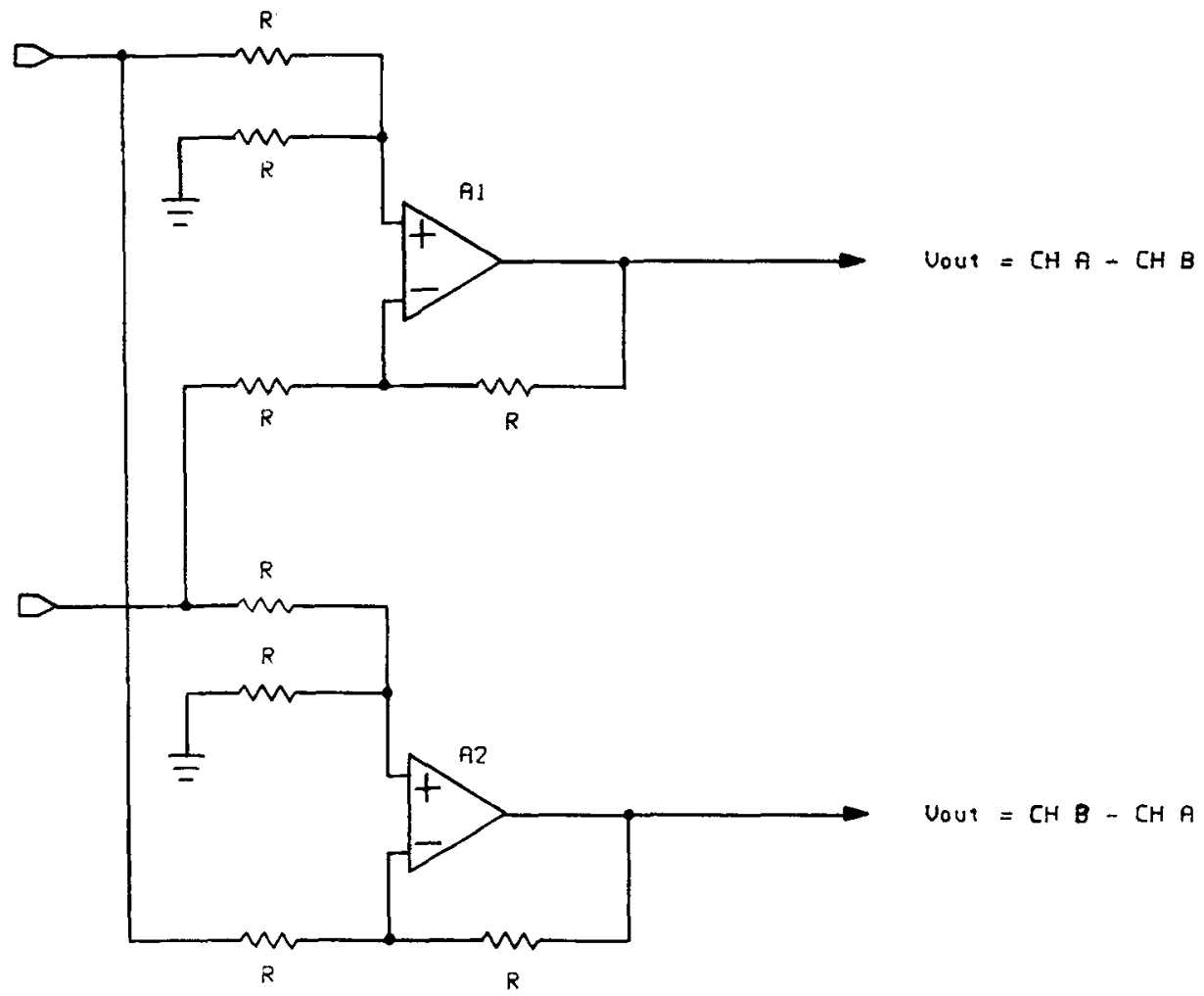

Figure 6. Function amplifiers configured for differentiation.

CH A Grating Dota

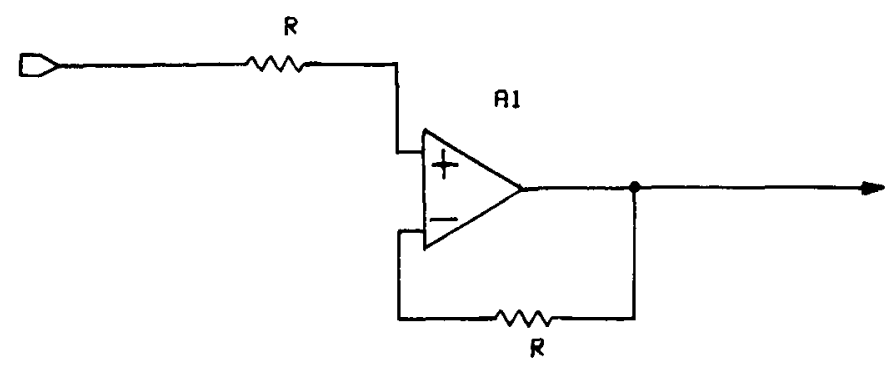

CH A Groting Doro

CH B Groting Doto

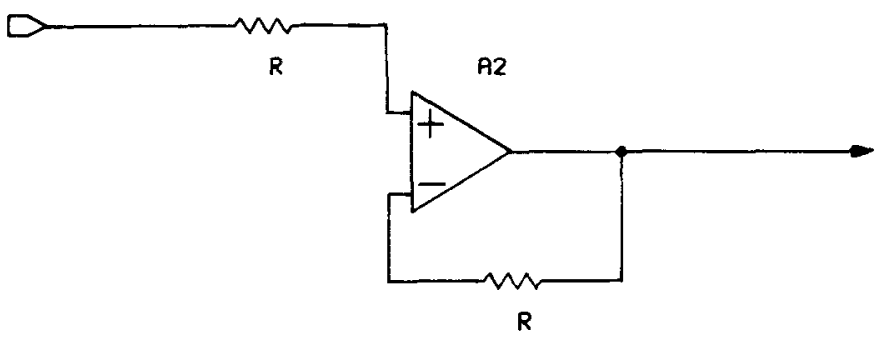

CH B Grating Doto

Figure 7. Function amplifiers configured for straight-through signals. 


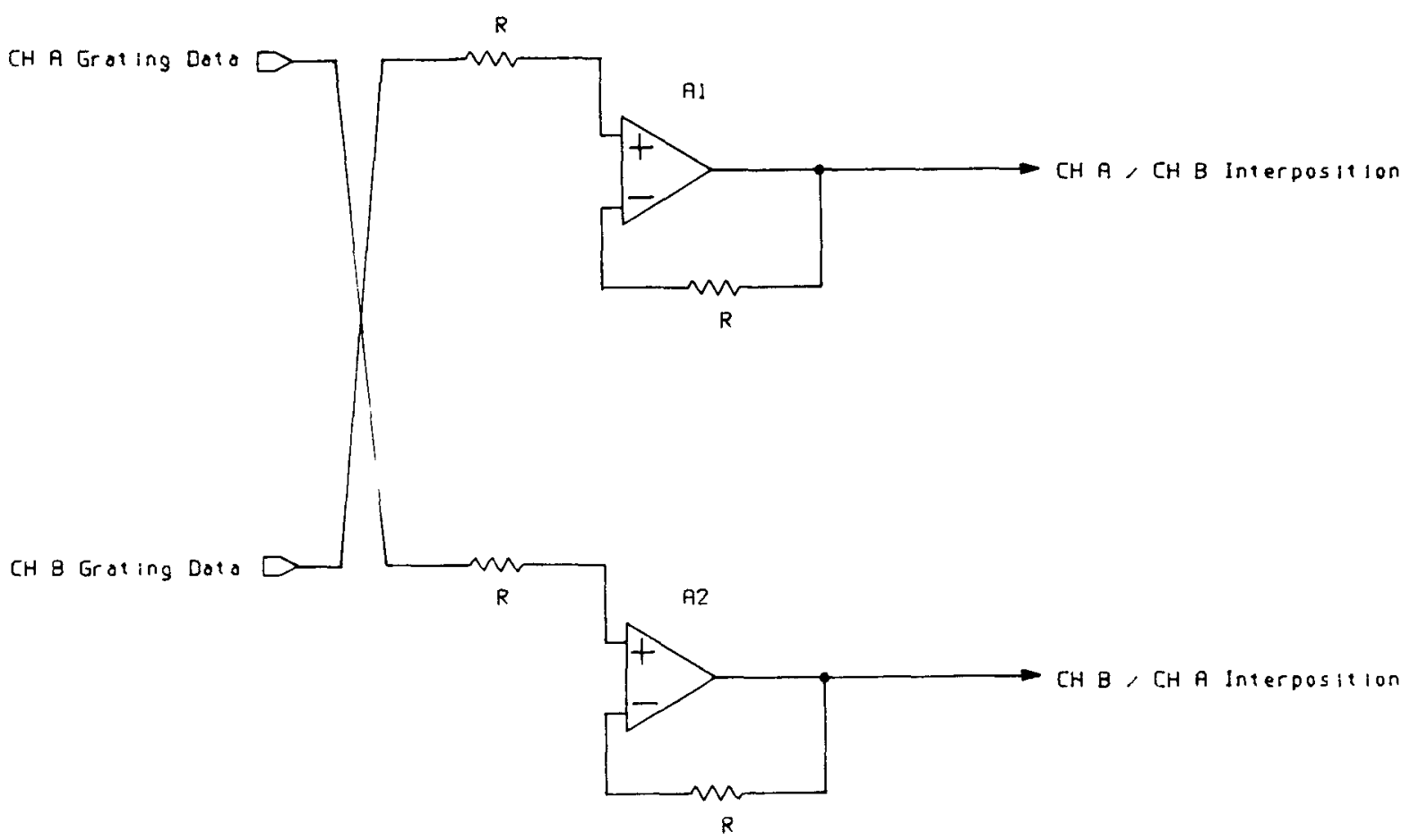

Figure 8. Function amplifiers configured for interposition. Data on Channel A output on Channel $B$ and data on Channel B output on Channel $A$.
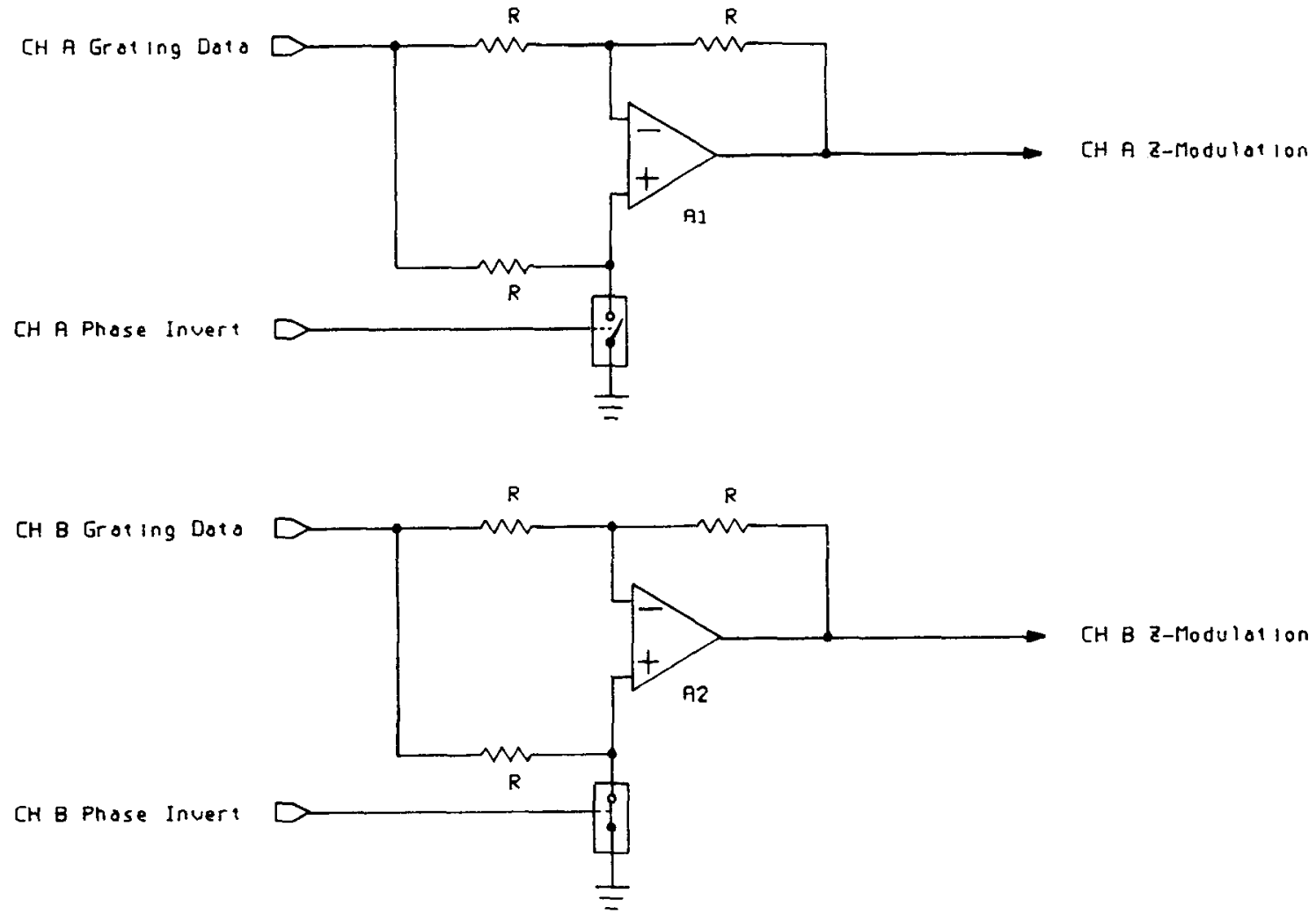

Figure 9. Operational amplifier for phase-inverting signals. Note that in the top part of the figure, the grating data on Channel $\mathbf{A}$ is not inverted. However, in the bottom part of the figure, the switch closer has activated phase inversion and the signal on Channel $B$ is consequently inverted. 
rapidly, this technique emulates the technique of continuous adjustment of contrast using a potentiometer. However, in the present design, these adjustments can be made under computer control, thus eliminating the need for an external circuit for contrast manipulations (Eskew, Pace, \& Rinalducci, 1984). In most applications, changes in mode or function will be made between frames in order to set up the appropriate stimulus conditions for the next presentation or trial.

The component nature of the design of the waveform generator allows for the introduction of an external device that can be used to provide external analog inputs to the DASP board in place of the signals from the DDAC board. The waveform generator may be used with a white noise (or bandpass filtered noise) generator to study the response of the visual system to a wider variety of stimuli. In this example, the white noise generator would be used to produce analog signals, and a possible application would be to study the detection of sinusoidal gratings in white noise. This would involve generating a white noise source on one channel from an external signal generator, presenting a grating on the other channel by means of the programmable waveform generator, and summing the output of the two channels. Another possible applilcation of the waveform generator, given the nature of the analog output, which ranges from 0 to a maximum of $\pm 5 \mathrm{~V}$, would be to use the analog output voltage to drive other equipment. A possible configuration illustrating this concept is outlined in Figure 10. In this example, the analog output voltage from one channel (Channel $A$ ) is used to sequentially read out voltage values from memory over Channels $A$ and $B$ at a continuous rate. The analog output voltage from one channel is used to drive a voltagecontrolled oscillator, which is used to produce gratings that can differ in spatial frequency. The other channel (Channel B)modulates a voltage-controlled gain amplifier, which is used to control contrast. In this case our device could be used to control these function generators to permit the study of temporal properties of the visual system with grating stimuli. For example, contrast could be varied over time so that gratings could be presented with various kinds of temporal envelopes at a very high rate under program control. This could be used to control the rate at which a grating is turned on and/or off. The influence of various kinds of onset and offset functions, such as linear or exponential ramps, could be examined in this way.

\section{CLI}

The conceptual diagram of the CLI board is presented in Figure 11. This board provides buffering between the computer and the DASP board. Its primary function is to allow for the selection of computer-controlled or manually controlled functions on the DASP board.

The CLI board provides the signals for selecting the functions or modes of stimulus presentation on the DASP board through a dual 3-bit control line. In addition, these functions may be manually set by dip switch configurations. Manual operations may be mixed with computer control. For example, Channel A may be operated manually, while the functions for Channel B may be programmed under computer control. Again, this opens a wide realm of possible configurations.

\section{SOME PROGRAMMING CONSIDERATIONS}

In the single-channel mode, gratings can be produced with our device in much the same way as that described

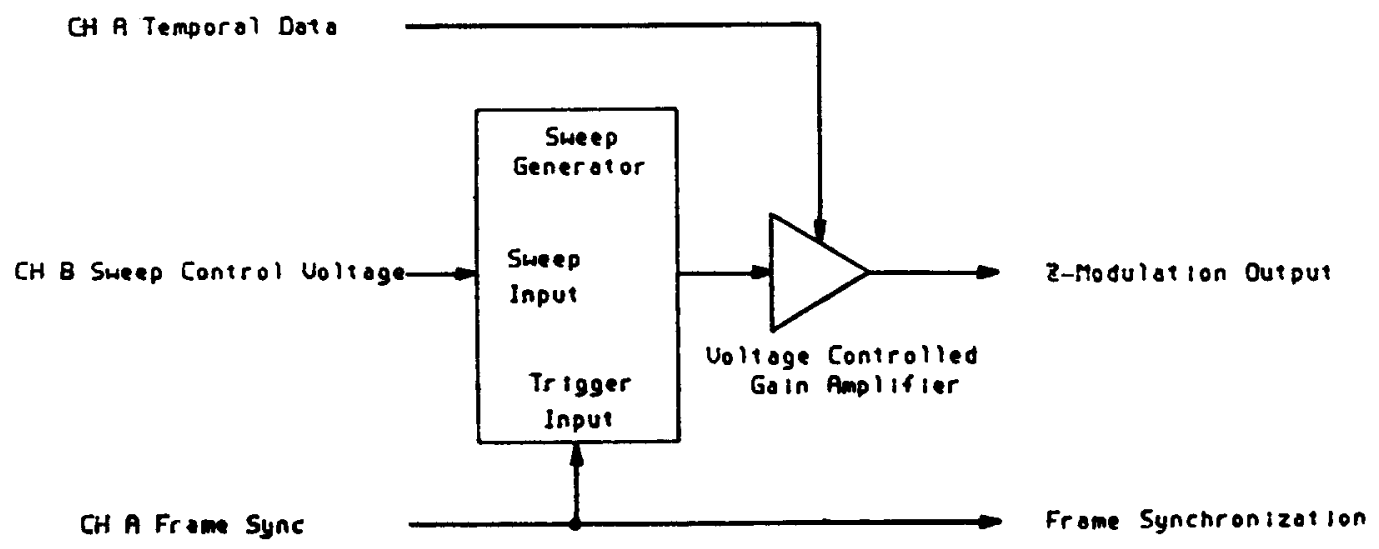

Uerical Scon Generator

Uertical Scan Modulation

Figure 10. Visual pattern stimulator configured to drive a sweep generator and a voltage-controlled amplifier for producing various temporal waveforms. 
by Fritsch and Keck (1978). Due to the hardware implementation of contrast scaling, contrast values can be simply selected from memory or input by the operator and latched prior to grating presentation. Thus, our software implementation for grating generation bypasses the need for a contrast scaling routine. Under this mode of stimulus presentation, the user has the option to use the $50-100-\mathrm{Hz}$ clock in the manner described by Fritsch and Keck (1978) as a means of synchronizing the output of the grating data with the sweep of the CRT. In order to fully realize the dual channel application of our pattern generator, this feature is not required. Before fully discussing this aspect of software development, we will focus on concepts involved in the simultaneous presentation of gratings on Channels A and B (it should be noted that the patterns may differ in a number of features, such as spatial, temporal, and contrast values).

Let us first consider the way in which data must be transferred from look-up tables in computer memory to the waveform generator in order to produce the grating stimuli on Channel A and Channel B to separate CRTs. The generation of two simultaneous gratings requires some form of interleaving, in which data is sampled al- ternately from each master table. Figure 12 shows the temporal sequencing of the various signals to produce interleaving. First the contrast data for Channel $A$ is written to the 8-bit VIA APA port of the SYM computer. Second, a control signal is necessary to latch this value. This is accomplished by writing to the appropriate control bit on the APB port that latches Channel A contrast. The latching operation involves the strobing or toggling of this bit. Similarly, the contrast value for Channel B is written to the APA port and latched by toggling the appropriate APB control bit. Changes in contrast over trials for one or both ports are accomplished by repeating some or all of the above steps.

As mentioned earlier, in the procedure used by Fritsch and Keck (1978) to produce gratings, a $50-\mathrm{Hz}$ external synchronization clock is utilized for program sequencing. Inherent in this approach are processor wait states. That is, data cannot be written to the VIA until an external synchronization pulse is received. Consequently, the central processing unit (CPU) is dedicated to reading the appropriate port for a sync pulse. In order to make more efficient use of the CPU, we have opted to use an interrupt-driven approach.

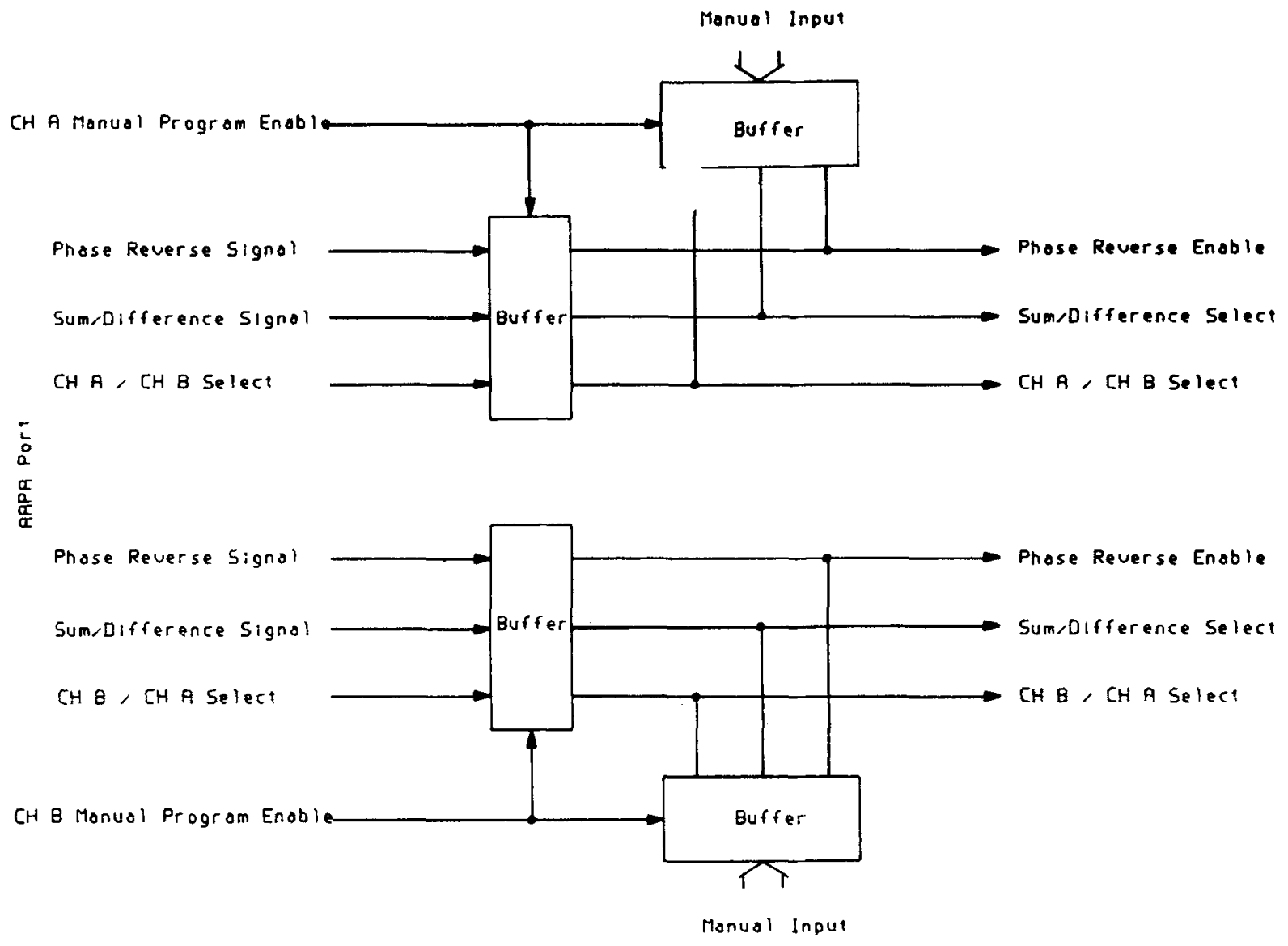

Figure 11. Control logic interface (CL.I). The interface between the SYM microprocessor and the manual inputs for producing the various functions of summation, differentiation, etc., are shown. 


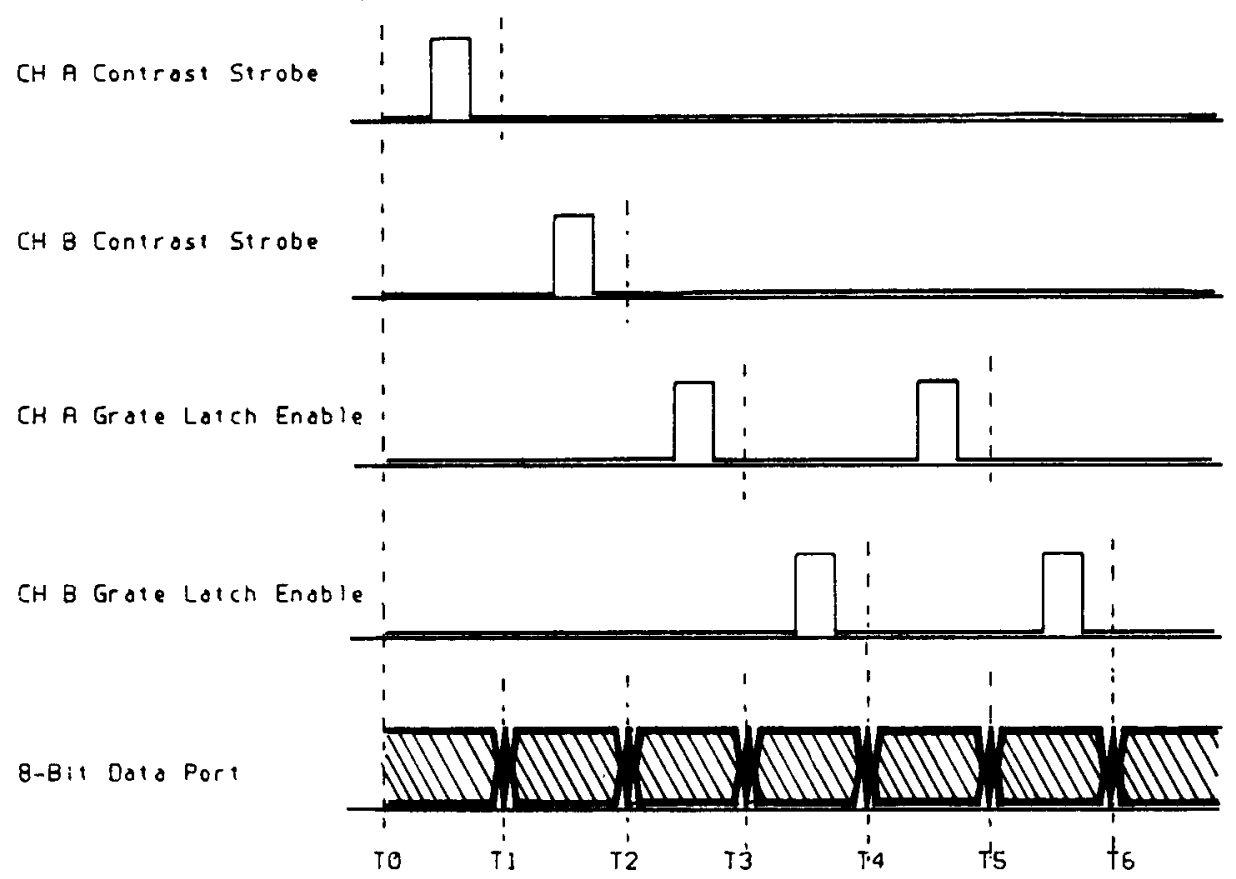

Figure 12. Temporal sequencing of control, contrast, latching, and data signals (see text for description).

We use the SYM's VIA ports, which contain two 16bit binary counters to provide the interrupt signals used by the CRT for frame synchronization. Timer 1 of the VIA is used in the one-shot mode to count down to zero, using the Phase 2 clock. When the timer counts down to zero, it issues an interrupt signal to the CPU. At that time we can change stimulus conditions and/or check for responses from the user. The value selected to load the $\mathrm{T} 1$ timer represents the number of clock cycles required to read through the two grating tables. This method leaves the CPU dedicated to presenting the grating values to the output port. We know from our previous computations during software design that at the end of one grating presentation the processor will receive an interrupt request. Thus, in contrast to Fritsch and Keck's (1978) design, in which frame synchronization is dependent on an externally generated input clock signal, our design provides an internally generated synchronization signal. This signal is generated when the T1 timer counts down from a preselected value to zero. At this time an interrupt signal is generated, reflecting completion of a grating frame. The PB7 port of the VIA is set during the interrupt interval. This signal is used as input to the $x$-axis (time base) of the CRT. Thus, the CPU need not wait for a sync signal to initiate a new grating presentation; the sync signal is provided to the external device, namely the $x$-axis of the CRT. This method reduces the processor time dedicated to monitor a port for an external sync signal, so that the CPU can be utilized for other functions.

In summary, we have described a relatively low-cost, flexible means of presenting patterns on a CRT. The design allows for a number of options, such as the presentation of gratings as well as nonstandard stimuli (white noise), the summation and differentiation of outputs to create complex luminance waveforms, and the switching of information from one channel to the other. In addition, our visual pattern stimulator permits a number of temporal options, such as phase reversals or the rate at which a stimulus is turned on and/or off (i.e., ramping). This device incorporates a hardware means of scaling grating data for contrast level, which consequently conserves computer memory. Finally, our procedure for producing synchronization signals, which uses internally generated interrupt signals, allows us to incorporte a number of software options, such as keyboard scanning and reaction timing, without significant time losses.

\section{REFERENCES}

ARnold N. D., \& HASTings, L. (1984). A bit-addressable I/O interface for microcomputers. Behavior Research Methods, Instruments, \& Computers, 16, 135-136.

Brown, C. R. (1977). A digital technique for generating moving grating patterns on an oscilloscope. Vision Research, 17, 299-300.

CAMPBELl, F. W., \& GreEN, D. G. (1965). Optical and retinal factors affecting visual resolution. Joumal of Physiology, 181, 576-593.

Eskew, R. T., JR., PACE, M., \& RinalducCi, E. J. (1984). A simple circuit for grating contrast adjustment. Behavior Research Methods, Instruments, \& Computers, 16, 538-539.

FritsCH, K., \& KECK, M. J. (1978). Grating generation by microcomputer. Vision Research, 18, 1083-1086.

Graham, N. (1972). Spatial frequency channels in the human visual system: Effects of luminance and pattern drift rate. Vision Research, 12, 53-68

Graham, N., \&achmias, J. (1971). Detection of grating patterns containing two spatial frequencies: A comparison of single channel and multiple channel models. Vision Research, 11, 251-259.

ROGERS, B. J. (1976). A technique for generating moving visual stimuli on a CRO. Vision Research, 16, 415-417. 